This item was submitted to Loughborough's Research Repository by the author.

Items in Figshare are protected by copyright, with all rights reserved, unless otherwise indicated.

\title{
A virtual inertia control strategy of interlinking converters in islanded hybrid AC/DC microgrid
}

\author{
PLEASE CITE THE PUBLISHED VERSION
}

https://doi.org/10.1109/ECCE.2019.8912225

PUBLISHER

IEEE

VERSION

AM (Accepted Manuscript)

\section{PUBLISHER STATEMENT}

(c) 2019 IEEE. Personal use of this material is permitted. Permission from IEEE must be obtained for all other uses, in any current or future media, including reprinting/republishing this material for advertising or promotional purposes, creating new collective works, for resale or redistribution to servers or lists, or reuse of any copyrighted component of this work in other works.

\section{LICENCE}

\section{All Rights Reserved}

\section{REPOSITORY RECORD}

Xiao, Jingyi, Alian Chen, Zhengyu Lin, and Haihua Xue. 2019. "A Virtual Inertia Control Strategy of Interlinking Converters in Islanded Hybrid AC/DC Microgrid". figshare. https://hdl.handle.net/2134/38220. 


\section{A Virtual Inertia Control Strategy of Interlinking Converters in Islanded Hybrid AC/DC Microgrid}

\author{
$1^{\text {st }}$ Jingyi Xiao \\ School of Control Science \\ and Engineering \\ Shandong University \\ City, Country \\ 201734481@mail.sdu.edu.cn
}

\author{
$2^{\text {nd }}$ Alian Chen \\ School of Control Science \\ and Engineering \\ Shandong University \\ Jinan, China \\ chenalian@sdu.edu.cn
}

\author{
$3^{\text {rd }}$ Zhengyu Lin \\ School of Mechanical, Electrical \\ and Manufacturing Engineering \\ Loughborough University \\ Loughborough, UK \\ z.lin@ieee.org
}

\author{
$4^{\text {th }}$ Haihua Xue \\ School of Control Science \\ and Engineering \\ Shandong University \\ Jinan, China \\ haihuaxue@mail.sdu.edu.cn
}

\begin{abstract}
In an islanded hybrid AC/DC microgrid, the existing intermittent distributed generations (DGs) and local loads variation frequently cause power fluctuation. And the DC bus voltage is sensitive to it. A virtual inertia control strategy of interlinking converters (ILCs) in an islanded hybrid microgrid is proposed in this paper, which restrains the DC bus voltage fluctuation and enhances the inertia of the hybrid microgrid. Traditional droop control methods of ILCs mostly only focus on power sharing. The proposed control strategy can not only maintain proportional power distribution between $\mathrm{DC}$ and $\mathrm{AC}$ subgrids, but also regulate the DC bus voltage directly. It can improve microgrid stability during DC-side loading transitions or distributed energy fluctuations. Moreover, no additional energy storage or inverters are required in a cost-effective manner. The validity of the proposed control method is verified by offline time-domain simulation in MATLAB/Simulink and real-time experiment in OPAL-RT digital platform.
\end{abstract}

Index Terms-Virtual inertia control, DC voltage stability, virtual synchronous machine (VSM), proportional power sharing, interlinking converters (ILCs), hybrid AC/DC microgrid.

\section{INTRODUCTION}

Nowadays, DC distributed generations (DGs), such as photovoltaics (PVs) and fuel cells (FCs), develop rapidly. Other DC systems, such as energy storages and DC loads like electric vehicles, gain more and more attention as well [1]. As a result, in recent years DC microgrids have gained an increasing interest. However, in consideration of the existing $\mathrm{AC}$ power systems, hybrid AC/DC microgrids have been considered as a cost-effective and efficient solution for future distribution network [2]. Fig. 1 shows the structure of a typical hybrid AC/DC microgrid. DGs are connected to AC and DC buses respectively, along with storage elements and $\mathrm{AC}$ and $\mathrm{DC}$ loads. Interlinking converters (ILCs) linked to the two buses are bridges between AC and DC subsystems.

A hybrid microgrid can operate in islanded mode or ongrid mode, depending on whether or not the point of common coupling (PCC) is kept open. ILCs coordinate the dual subsystems and exchange power proportionally between them and regulate $\mathrm{AC}$ and $\mathrm{DC}$ bus voltages stability. Considering the low Inertia of the intermittent DGs, microgrids often suffer

This work has received funding from the Royal Society International Exchanges (IE161121) of the U.K and the National Natural Science Foundation of China (51877128).

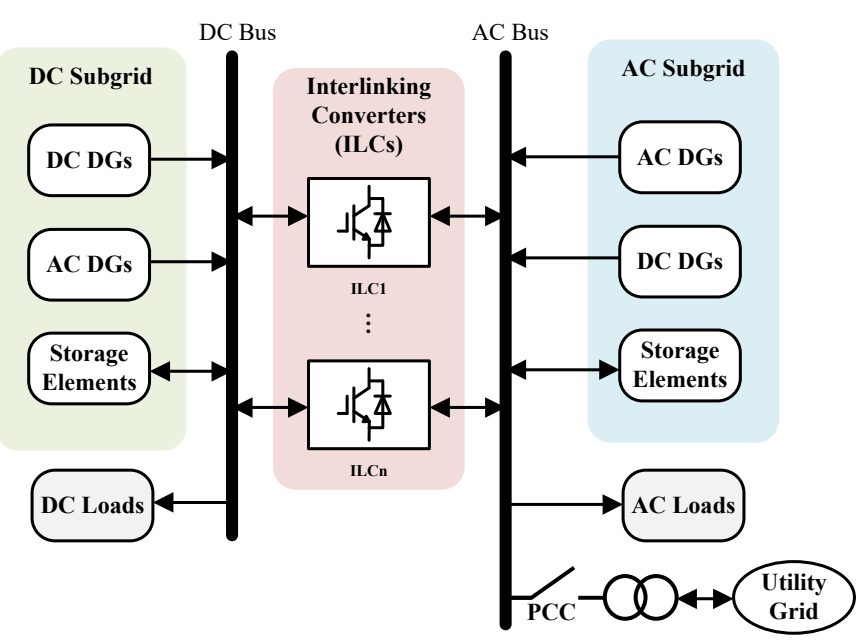

Fig. 1. Structure of hybrid AC/DC microgrid.

from stability problems. ILCs play a crucial part in the control of hybrid microgrids, especially in islanded mode when the system lacking the support of utility grid.

The existing control methods of ILCs can be generally divided into two categories: communication-less methods and communication-based methods [3]. Those communicationbased methods are able to manage power flows accurately by collecting and exchanging essential local data through communication lines [4], [5]. Nevertheless, the existing of communication systems may reduce the reliability of the microgrid because any communication failure can lead to instability of the whole microgrid. Therefore, communicationless methods, such as traditional droop control strategies, are widely adopted, due to their simpler structure and higher reliability [6].

A communication-less method in [6] and [7] normalized the droop control of the AC-DC converter in the AC side and DC side, and can achieve proportional power sharing by keeping the per-unit frequency and per-unit DC voltage to the same point. A uniform control strategy based on hierarchical method is reported in [8].However, these two methods do not discuss how to enhance the inertia and stability of DC bus.

Several recent researches involve how to increase system inertia, but the existing virtual inertia control methods usually 
focus on the $\mathrm{AC}$ networks, such as virtual synchronous machine (VSM) technology [9]. In [10] and [11], the concept of synchronverter is proposed. By applying proper control methods over the power electronics converters systems, mechanical and electrical characteristics of the synchronous machine (SM) can be emulated. In [3] and [12], the dynamic frequency stability of AC subgrid is discussed. Reference [13]-[15] analyze the VSM control methods applied in DGs.

In the matter of DC networks, in order to restore the DC bus voltage, the improved droop control method in [16] also needs a low-bandwidth communication, which reduces its reliability. In [9] and [17], VSM technology in DC microgird is mentioned but without considering the power sharing function of ILCs in hybrid microgirds.

To address the above-mentioned drawbacks, in this paper, a virtual inertia control strategy of ILCs is proposed, in order to improve DC subgrid voltage inertia and stability based on normalized droop control method. This method can not only maintain proportional power distribution between $\mathrm{DC}$ and $\mathrm{AC}$ subgrids, but also regulate the DC subgrid voltage directly and increase virtual inertia without any additional energy storage or inverters. It makes the ILCs appearing to have high inertia in order to suppress DC voltage disturbance during DC-side loading transitions and distributed energy fluctuation.

\section{Autonomous Operation Within Subgrids}

As mentioned above, a typical islanded hybrid microgrid consists of AC and DC subgrids. This section reviews the droop control method applied in AC subgrids, and analyzes the similar control method within DC subgrids. The autonomous operation theories within subgrids are significant because they are the preconditions for deriving the ILCs control strategy in the next section. In addition, since the main subject of this paper is ILCs connecting AC and DC buses, only active power control method is discussed here.

\section{A. Droop Control for AC Subgrid}

Droop control applied to an AC subgrid with at least two paralled DGs has widely been investigated [6]. As shown in Fig. 2, locally measured active power $f_{a c}$ is relevant to the references of AC voltage frequency. Upon reaching steady state, without considering line impedance, these paralleled ACDGs connected to the AC bus come to a common frequency

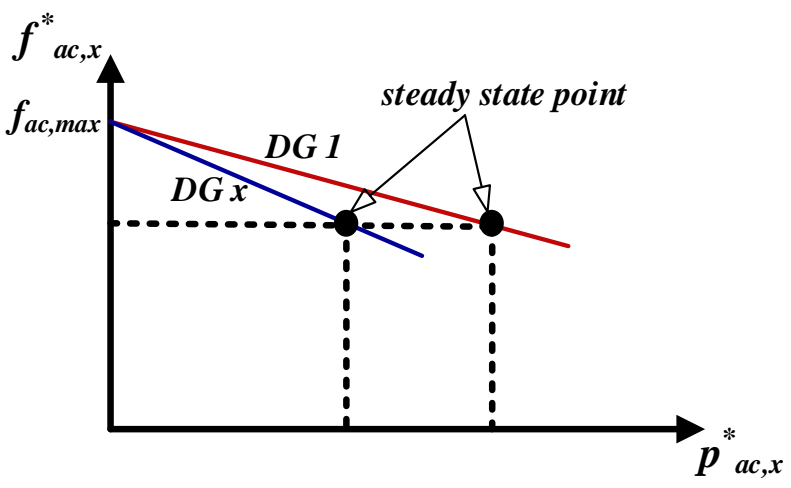

Fig. 2. $P-f$ droop characteristics in AC subgrid. autonomously. The $P-f$ droop equation of the $x$ th DG connected to $\mathrm{AC}$ bus can be appropriately written as:

$$
\begin{gathered}
f_{a c, x}^{*}=f_{a c_{-} \max }-m_{a c, x} p_{a c, x} \\
m_{a c, x}=\left(f_{a c_{-} \max }-f_{a c_{-} \min }\right) / P_{a c_{-} \text {rated }, x}
\end{gathered}
$$

where $f_{a c_{-} \max }$ is the maximum AC voltage frequency at no load and $f_{a c_{-}}$min is the minimum permissible frequency at rated load, respectively. $P_{a c_{-} \text {rated, } x}$ is the rated power of the $x$ th DG. $m_{a c, x}$ is the droop coefficient of the $x$ th DG and is a positive number, representing the absolute value of the gradient of the lines (negative) shown in Fig. 2.

Since $f_{a c_{-} \max }$ and $f_{a c_{-} \min }$ are the same in the same subgrid, (3) can be inferred from (2):

$$
\begin{aligned}
m_{a c, 1} P_{a c_{-} \text {rated }, 1} & =m_{a c, 2} P_{a c_{-} \text {rated }, 2} \\
& =\cdots \\
& =m_{a c, x} P_{a c_{-} \text {rated }, x}
\end{aligned}
$$

As a result, in order to make sure all the AC-DGs share the power demand in proportional to their rated power, the droop coefficients should be tuned according to (3).

\section{B. Droop Control for DC Subgrid}

In DC subgrid, attention only should be paid to these two quantities: DC bus voltage and active power. Similar with the droop characteristics in AC subgrid, DC bus voltage falls linearly with the increase of output active power, as shown in Fig. 3. Ignoring line impedance, DC-DGs connected to the DC bus reach the same voltage at the steady-state point. The $P-u_{d c}$ droop equation of the $y$ th DG connected to the DC bus is given by:

$$
\begin{gathered}
u_{d c, y}^{*}=U_{d c_{-} \max }-n_{d c, y} p_{d c, y} \\
n_{d c, y}=\left(U_{d c_{-} \max }-U_{d c_{-} \min }\right) / P_{d c_{-} \text {rated }, y}
\end{gathered}
$$

where $U_{d c_{-} \text {max }}$ is the maximum DC voltage at no load and $U_{d c_{-} \text {min }}$ is the minimum permissible DC voltage at rated load, respectively. $P_{d c_{-} \text {rated, } y}$ is the rated power of the $y$ th DG. $n_{d c, y}$ is the droop coefficient of the $y$ th DG and is a positive number, representing the absolute value of the gradient of the lines (negative) shown in Fig. 3.

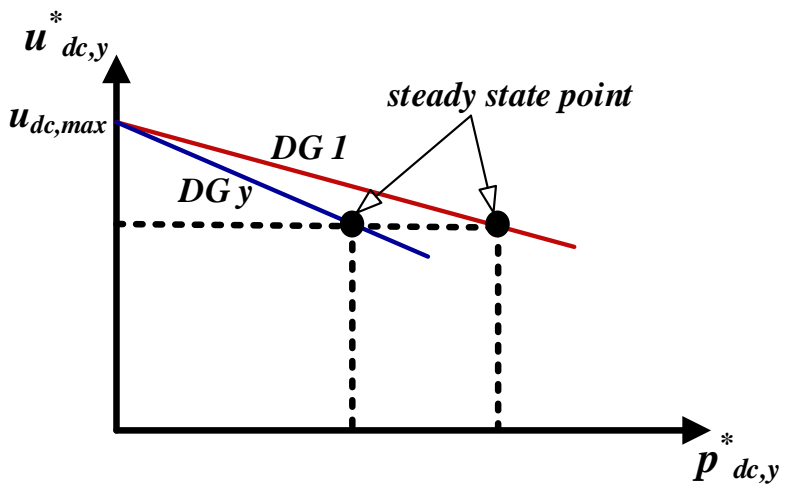

Fig. 3. $P-u_{d c}$ droop characteristics in DC subgrid. 
Since $U_{d c_{-} \max }$ and $U_{d c_{-} \text {min }}$ are the same in the same subgrid, it can be inferred from (5):

$$
\begin{aligned}
n_{d c, 1} P_{d c_{-} \text {rated }, 1} & =n_{d c, 2} P_{d c_{-} \text {rated }, 2} \\
& =\cdots \\
& =n_{d c, y} P_{d c_{-} \text {rated }, y}
\end{aligned}
$$

As a result, in order to make sure all the DC-DGs share the power demand in proportional to their rated power, the droop coefficients should be tuned according to (6).

\section{IMPROVED CONTROL STRATEGY OF ILCS}

Proportional power sharing must be realized throughout the whole AC/DC hybrid microgrid. And this function is often realized by the control of ILCs, the bridges between AC and DC buses. Besides, considering high penetration rate of lowinertia inverter-interfaced DGs, especially in the DC subgrid, it is also significant to guarantee the DC bus voltage stability during load transitions and distributed energy fluctuations. Non-schedulable DGs, such as PVs in maximum power point tracking (MPPT) mode, aggravate the stability issue.

As a result, a novel control strategy of ILCs is proposed in this section, which can directly regulate the DC bus voltage to realize proportional power distribution between two subgrids. What's more, It can improve the dynamic stability of microgid as well, without any additional storage elements.

As shown in Fig. 4, the research object, ILCs, apply the traditional six-switch bidirectional converter topology, with a LC filter. The ILCs are controlled to operate in voltage source mode, in order to provide voltage support for the DC bus. During the operation of ILCs, instantaneous three phase voltage and current $\left(u_{a b c}, i_{a b c}\right)$ are measured at $\mathrm{AC}$ side and DC voltage and current $\left(u_{d c}, i_{o}\right)$ are measured at DC side. Fig. 4 also reveals that the improved control strategy includes two key steps: Direct DC Voltage Control and Virtual Inertia Control. Their principles and functions are describes in detail in this section.

\section{A. Proportional Power Sharing Technology}

As mentioned in section 2, because most DGs adopt droop control, the DC voltage variation and AC frequency variation are linearly related to the output active power respectively. Therefore, the DC side and AC side, as a whole, appear droop characteristics as well. In other words, the frequency variation reflects the output active power of $\mathrm{AC}$ subgrid and the DC voltage variation reflects the output active power of DC subgrid.

However, frequency variations and DC voltage variation happen in two independent subsystems, and cannot be unified unless being brought to a common per unit range. The simple normalized droop control scheme in [6] shows a normalized droop control method. According to (1), (2), (4) and (5), the normalized equations is obtained as:

$$
\left\{\begin{aligned}
f_{p u} & =\frac{f_{a c}^{*}-0.5\left(f_{a c_{-} \max }+f_{a c_{-} \min }\right)}{0.5\left(f_{a c_{-} \max }-f_{a c_{-} \min }\right)} \\
u_{d c, p u} & =\frac{u_{d c}^{*}-0.5\left(U_{d c_{-} \max }+U_{d c_{-} \min }\right)}{0.5\left(U_{d c_{-} \max }-U_{d c_{-} \min }\right)}
\end{aligned}\right.
$$

where $f_{p u}$ and $f_{a c}^{*}$ are the per unit frequency and instantaneous frequency of AC subgrid, respectively. $u_{d c, p u}$ and $u_{d c}^{*}$ are the per unit voltage and instantaneous voltage of DC subgrid, respectively.

As long as keeping $f_{p u}=u_{d c, p u}$, the whole active power demand can be shared between AC and DC subgrids according to their rated power. That is to say, the whole active power demand can be shared among all AC-DGs and DC-DGs, according to their rated power.
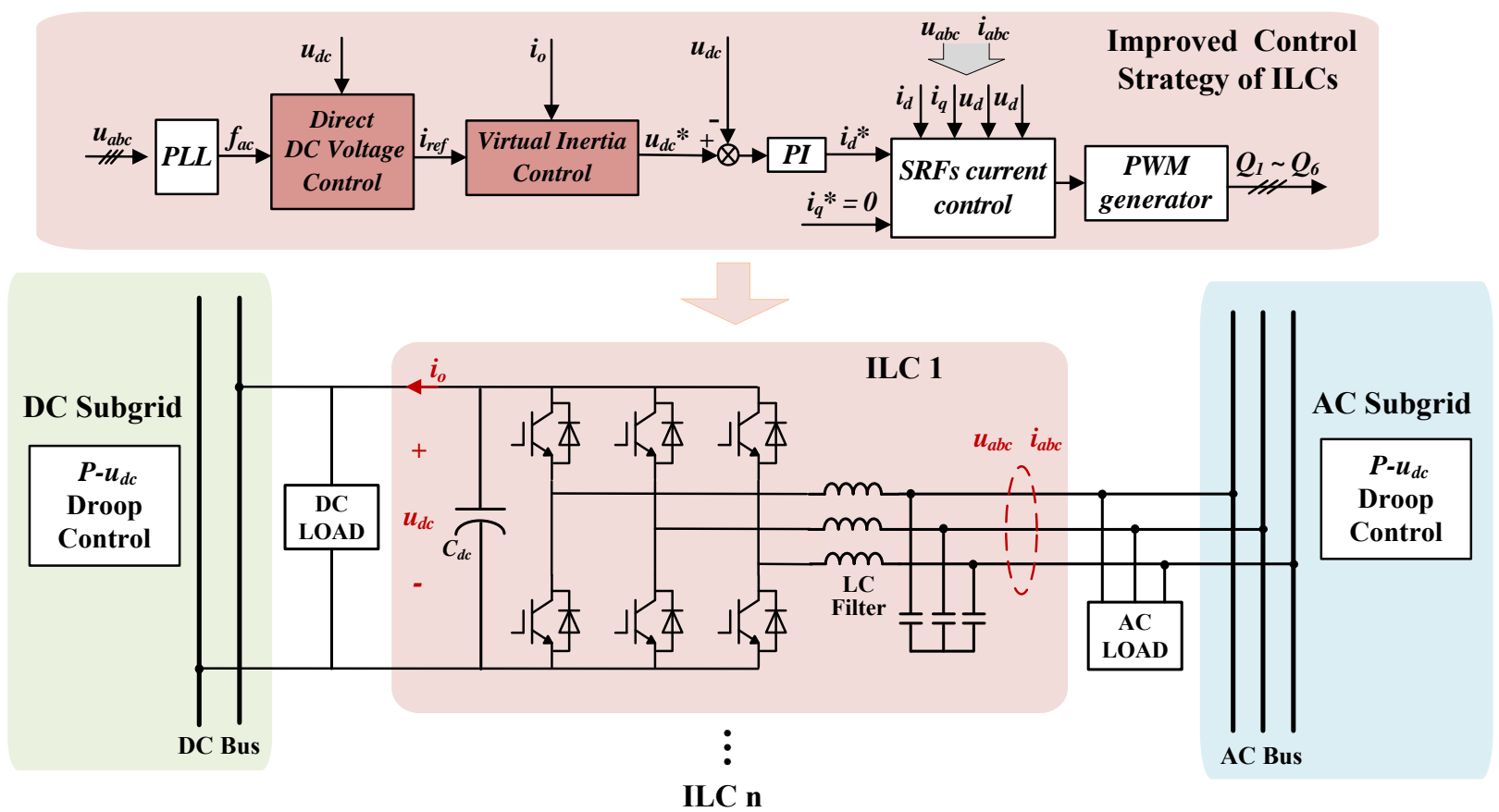

Fig. 4. Block diagram of ILCs control strategy in an islanded hybrid microgird. 
However, traditional method usually use $\left(f_{p u}-u_{d c, p u}\right)$ in their control loop, which can only realize proportional power sharing but not offer voltage support to DC bus.

As a consequence, the proposed improved control strategy adopts a direct DC voltage control to improve DC voltage response. According to (1), (2), (4) and (5), the droop characteristic of AC and DC subgrids can be unified in a novel way. Setting AC and DC instantaneous active power to be equal, real-time dynamic reference $U_{d c}$ can be revised as:

$$
\begin{gathered}
u_{d c_{-} r e f}=u_{d c}^{*}=U_{d c_{-} \max }-k_{f-u}\left(f_{a c_{-} \max }-f_{a c}^{*}\right) \\
k_{f-u}=\frac{\left(U_{d c_{-} \max }-U_{d c_{-} \min }\right) P_{a c_{-} \text {rated }}}{\left(f_{a c_{-} \max }-f_{a c_{-} \min }\right) P_{d c_{-} \text {rated }}}
\end{gathered}
$$

As long as keeping the instantaneous DC-side voltage $u_{d c}^{*}$ to be equal with $u_{d c_{-} \text {ref }}$, the output active power of $\mathrm{AC}$ and DC subgrids will be naturally proportional to their rated power in steady state. In addition, Equation (8) and (9) show that the control of ILCs is also an improved droop control as well. The droop characteristics can be better explained by Fig. 5. In Fig. 5, $P_{I L C}$ is the active power transferred from DC side to AC side by ILCs. $e_{u d c}$ is the error of DC voltage $\left(e_{u d c}=u_{d c, r e f}-u_{d c}^{*}\right)$ and is limited to a small range from $e_{\min }$ to $e_{\max }$. There is a liner relationship between $e_{u d c}$ and $P_{I L C}$.

The control diagram of the proportional power sharing is shown in Fig. 6. kn is the droop coefficient of the $n$th ILC, which realizes the proportional power sharing among parallel ILCs in the form of of $k_{1} P_{1}=k_{2} P_{2}=\cdots=k_{n} P_{n}$. The integral term $1 J_{I L C} S$ is adopted to realize zero steady-state error control of $u_{d c}$ according to DC-side output current. The integral term also increases the DC voltage inertia the virtual

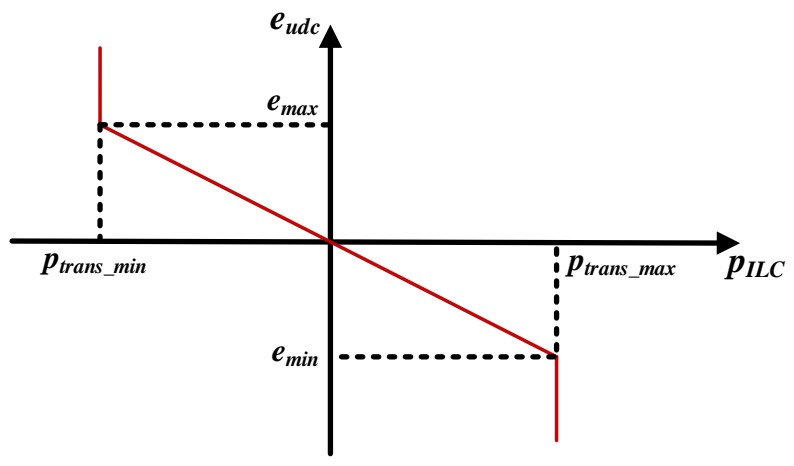

Fig. 5. $P-e_{u d c}$ droop characteristics in DC subgrid.

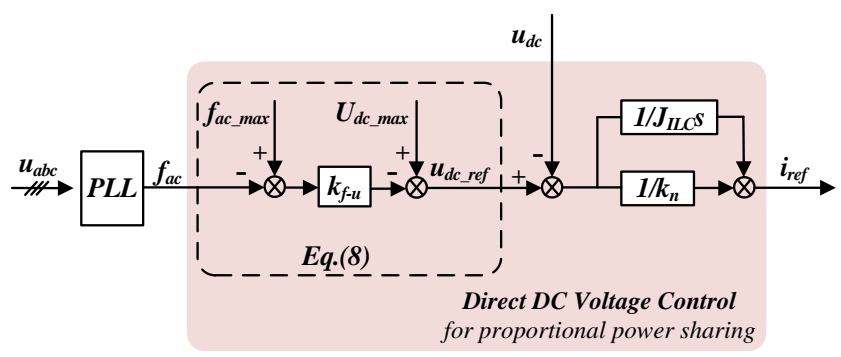

Fig. 6. Block diagram of proportional power sharing control. interrelating to damping coefficient $J_{I L C}$, which improves the dynamic DC voltage stability.

By adopting this direct DC voltage control, proportional power sharing as well as DC bus voltage support can be realized simultaneously.

\section{B. Virtual Inertia Technology}

In AC microgrids, the VSM technology is often used to emulate the inertia and damping characteristic of the SM, and realize its primary frequency regulation [9]. It is a kind of active power - angular frequency $(P-\omega)$ control. The mechanical equation of the VSM can be described as:

$$
P_{\text {set }}-P_{e}-D_{p}\left(\omega^{*}-\omega_{n}\right)=J \omega^{*} \frac{d \omega^{*}}{d t} \approx J \omega_{n} \frac{d \omega^{*}}{d t}
$$

where $J$ is the virtual inertia coefficient of the virtual rotor and $D_{p}$ is the virtual damping coefficient. omega $a_{n}$ and omega* are the rated angular frequency and actual angular frequency, respectively. $P_{\text {set }}$ and $P_{e}$ are the active power reference value and the electromagnetic power of the VSM.

When AC microgrid reaches its steady state, the angular frequency remains constant, so $d \omega^{*} / d t=0$, (10) can be written as:

$$
\omega^{*}=\omega_{n}+\frac{P_{s e t}}{D_{p}}-\frac{1}{D_{p}} P_{e}
$$

Equation (11) is actually an active power - angular frequency droop $(P-\omega$ droop) control:

$$
\begin{gathered}
\omega^{*}=\omega_{n o_{-} \text {load }}-m_{\omega} P_{e} \\
\omega_{\text {no_load }}=\omega_{n}+P_{\text {set }} / D_{p}, m_{\omega}=1 / D_{p}
\end{gathered}
$$

where $m_{\omega}$ is the droop coefficient of $P-\omega$ droop control. Comparing (1) and (12), it can be referred that $m_{\omega}=2 \pi m_{a c}$, since $m_{a c}$ is the droop coefficient of $P-f$ droop control mentioned in section 2 in equations (1), (2) and (3). Therefore, the VSM control actually can be seen as a modified droop control.

VSM technology used in the control of DG inverters can effectively improve the frequency stability of AC microgrids. Moreover, autonomous control in the DC subgrid is similar to that in AC subgrid, to some extent, in the respect of droop characteristics. Therefore, to address the stability issues of DC voltage, a virtual inertia control mimicking VSM can be applied.

In the DC subgrid, $P-u_{d c}$ droop control is widely adopted, as (4), (5) and (6) has illustrated. Since $P_{d c}$ approximately equals to $U_{n} i_{o}$, where $U_{n}$ is the rated DC bus voltage, (4) can be rewritten in the form of voltage-current droop $\left(u_{d c}-i_{d c}\right.$ droop) control, described as:

$$
\begin{gathered}
u_{d c}^{*}=U_{d c, \text { no-load }}-n_{i} i_{o} \\
n_{i}=U_{n} n_{d c}
\end{gathered}
$$

where $n_{i}$ is the droop coefficient of $u_{d c}-i_{d c}$ droop control and $n_{i}=U_{n} n_{d c}$ since $n_{d c}$ is the droop coefficient of $P-u_{d c}$ droop control mentioned in equations (3) and (4). 
Given the similarities between AC and DC droop control algorithms, according to (10), the virtual inertia equation of DC subgrid can be written as:

$$
I_{s e t}-i_{o}-D_{b}\left(u_{d c}^{*}-U_{n}\right)=C_{v} u_{d c}^{*} \frac{d u_{d c}^{*}}{d t} \approx C_{v} U_{n} \frac{d u_{d c}^{*}}{d t}
$$

where $I_{\text {set }}$ and $i_{o}$ are the DC output current reference and DC output actual current of the ILCs, respectively. $U_{n}$ is the rated DC bus voltage. $D_{b}$ is the damping coefficient, reciprocal of droop coefficient $n_{i}$ in (15). $C_{v}$ is the introduced virtual capacitance of ILCs, that is, the virtual inertia coefficient. It is worth noting that $C_{v}$ is related to the inherent storage element or capacity of the microgird, but not exactly equivalent to the DC-side capacitor of ILC.

Similarly, with that in the AC subgrid, the virtual inertia control in the DC subgrid is actually a modified $P-u_{d c}$ droop control, introducing virtual inertia and virtual damping into DC subgrid. This virtual inertia method can be applied in ILCs after the above-mentioned direct DC voltage control. Fig. 7 shows the block diagram of the virtual inertia control of ILCs.

According to (16), the loop including $1 / C_{v} U_{n}$ helps realizing the increase of virtual inertia. The loop including $D_{b}$ influences its sensitivity to the voltage deviation, which means the damping effect. The virtual inertia control part can enhance the inertia and stability of the DC bus by introducing the virtual inertia and virtual damping into the ILCs.

Fig. 8 is the detailed and complete control block diagram, include five parts: Phase-locked loop (PLL), direct DC voltage control (for proportional power sharing), virtual inertia control (for increasing DC bus inertia and stability), voltage and current dual-loop control and PWM generator.

The $U / I$ dual-loop consists of a PI voltage outer loop control to track DC bus voltage reference, and a current inner loop using the well-known decoupled current control based on synchronous reference frames (SRFs). After the DC voltage

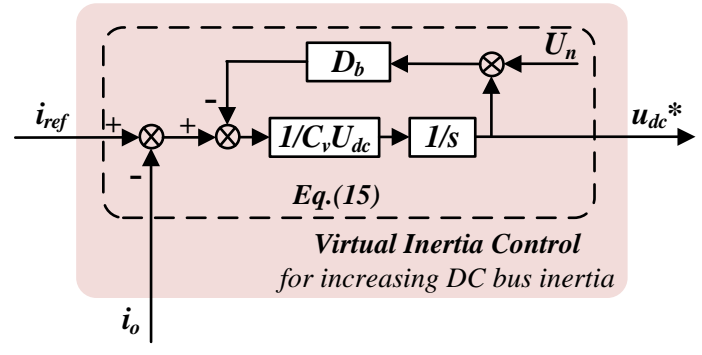

Fig. 7. Block diagram of virtual inertia control. reference value $u_{d c}^{*}$ is calculated, it is compared with the local measured actual DC voltage $u_{d c}$. The error then input into a PI controller, to get the reference value of current loop. The detailed discrimination of SRF current control can be found in [9]. Moreover, since there is no reactive power in the DC side, reactive current reference value $i_{q}^{*}$ is set to be zero during the operation of ILCs.

\section{Simulation AND EXPERIMENTAL VERIFICATIONS}

In this section, a time-domain simulation platform of a hybrid microgrid is built in Matlab/Simulink according to Fig. 9, in order to validate the functions of the proposed virtual inertial control scheme of ILCs. The DC subgrid including several DC-DGs is simulated by a bidirectional DC-DC converter connected to the DC bus. Similarly, the AC subgrid is mimicked by a bidirectional AC-DC converter connected to the AC bus. As is mentioned in section 2, the AC and DC subgrids are controlled by the $P-f$ droop control and $P-u_{d c}$ droop control methods respectively. The ILC and AC subgrid converter both adopt a traditional six-switch full bridge topology with an LC filter, as shown in Fig. 4.

The rated power of $\mathrm{AC}$ and DC subgrids are both $20 \mathrm{~kW}$. AC frequency is from $49 \mathrm{~Hz}$ to $51 \mathrm{~Hz}$ while DC bus voltage range is $590 \mathrm{~V}$ to $600 \mathrm{~V}$. System parameters of the hybrid microgrid is illustrated in Table. I. It can be observed that DC load demand has two modes, equal to $1.2 \mathrm{~kW}$ in light mode while $6.2 \mathrm{~kW}$ in heavy mode. Similarly, AC load demand

TABLE I

SYSTEM PARAMETERS OF HYBRID MICROGRID

\begin{tabular}{cc}
\hline \hline Global parameters & Value \\
\hline AC subgrid rated active power & $P_{d c, \max }=20 \mathrm{~kW}$ \\
DC subgrid rated active power & $P_{a c, \max }=20 \mathrm{~kW}$ \\
AC frequency range & $49 \mathrm{~Hz}<f<51 \mathrm{~Hz}$ \\
AC RMS voltage range & $215 \mathrm{~V}<v_{R M S}<225 \mathrm{~V}$ \\
DC voltage range & $590 \mathrm{~V}<U_{d c}<600 \mathrm{~V}$ \\
\hline Circuit parameters & Value \\
\hline ILC DC-side capacity & $C_{d c}=4700 \mu F$ \\
LC filter & $L_{f}=8 m \mathrm{H}, C_{f}=4700 \mu F$ \\
Line impedance & $L_{Z}=0.3 m H, R_{Z}=0.3 \Omega$ \\
DC load & $1.2 \mathrm{~kW}(\mathrm{Light}), 6.2 \mathrm{~kW}(\mathrm{Heavy})$ \\
AC load & $3.8 \mathrm{~kW}$ (Light), $8.8 \mathrm{~kW}$ (Heavy) \\
\hline Control parameters & Value \\
\hline Virtual inertia coefficient & $C_{v}=0.00167$ \\
Virtual damping coefficient & $D_{b}=10$ \\
\hline \hline
\end{tabular}

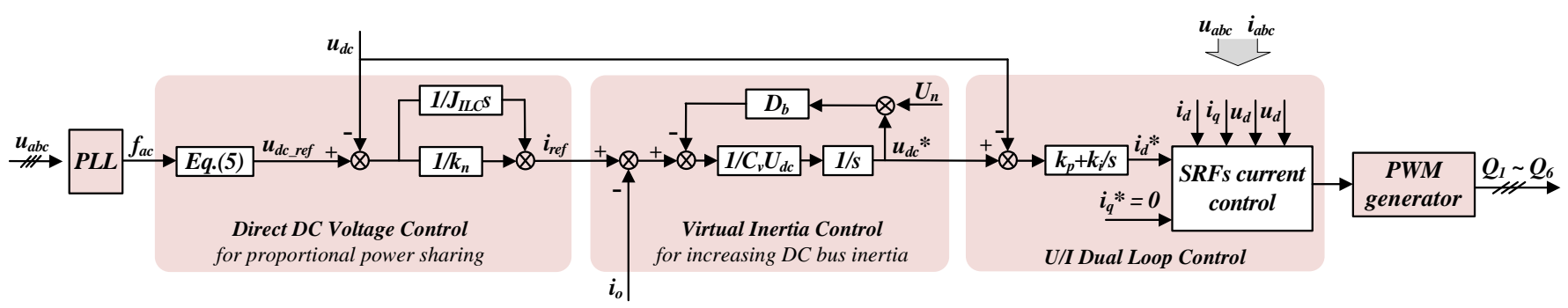

Fig. 8. Detailed block diagram of the proposed improved control strategy of ILCs. 


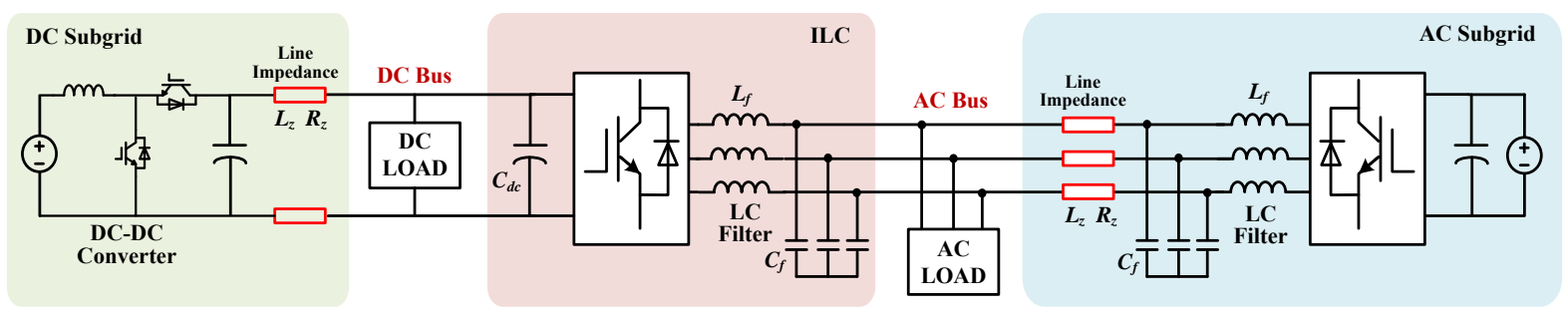

Fig. 9. Detailed structure of the tested hybrid AC/DC microgrid.

equals to $3.8 \mathrm{~kW}$ in light mode while $8.8 \mathrm{~kW}$ in heavy mode. The difference between light and heavy mode is $5 \mathrm{~kW}$. The performance of ILC can be verified by load transition process between two modes.

\section{A. Proportional Power Sharing}

Fig. 10 and Fig. 11 show the simulation results during load transitions. From $0 \mathrm{~s}$ to $1 \mathrm{~s}$ (Stage 1: $0 \mathrm{~s}$ to $1 \mathrm{~s}$ ), DC load is in light mode load while AC load is heavy. At $1 \mathrm{~s}$ (Stage 2: $1 \mathrm{~s}$ to $3 \mathrm{~s}$ ), DC load change to heavy mode while AC side remains unchanged, so total power demand increases $5 \mathrm{~kW}$. At $3 \mathrm{~s}$ (Stage 3: $3 \mathrm{~s}$ to $5 \mathrm{~s}$ ), AC load change to heavy mode while DC side remains heavy, so the total power demand increases $5 \mathrm{~kW}$, either.

In Fig. 10 (a), when reaching steady state, frequency falls about $0.25 \mathrm{~Hz}$ everytime the total load demand increases 5 $\mathrm{kW}$, no matter which side does it happened. In Fig. 10 (b), DC voltage both droops about $1.3 \mathrm{~V}$ at steady state in Stage 1 and Stage 3, corresponding to the load increase (5 $\mathrm{kW}$ both), either. As is discussed in in section 2, according to the AC side $P-f$ droop control and DC side $P$ - $u_{d c}$ droop control, frequency droops linearly due to AC load raising while DC voltage droops linearly due to DC load raising. As a consequence, Fig. 10 (a) and Fig. 10 (b) not only proves the droop control methods in both subgrids respectively, but also verified the effectiveness of the ILC, which can coordinate the power flows between AC and DC subgrids.

When focusing on the performance of ILC under these circumstances, Fig. 10 (c) shows the simulation results of ILC output DC current to DC side $\left(I_{o}\right)$, positive in rectification mode. In Stage $1, I_{o}$ is negative, which means ILC transfers power to from DC subgrid to AC subgrid, the ILC operates in rectification mode. This is because, at this moment, the power shortage is higher in AC side than in DC side. In Stage 2, DC load is heavier than AC load, so the ILC operates in inversion mode, transmitting power from AC subgrid to DC subgrid $\left(I_{O}\right.$ is positive). In Stage 3, after AC load sudden raises at $3 \mathrm{~s}, I_{o}$ droop from +2 to -2 rapidly and smoothly, and ILC operates in inversion mode again at steady state.

Fig. 11 shows the active power flows from Stage 1 to Stage 3. No matter in which stage, the output power of the dual subgrids are always approximately the same at steady states, equal to half of the total power demand, as shown in Fig. 11 (a) and Fig. 11 (b). As a result, Its verified to be effective for the ILC to manage proportional power flows, since the rated active power of AC and DC subgrids are the same.

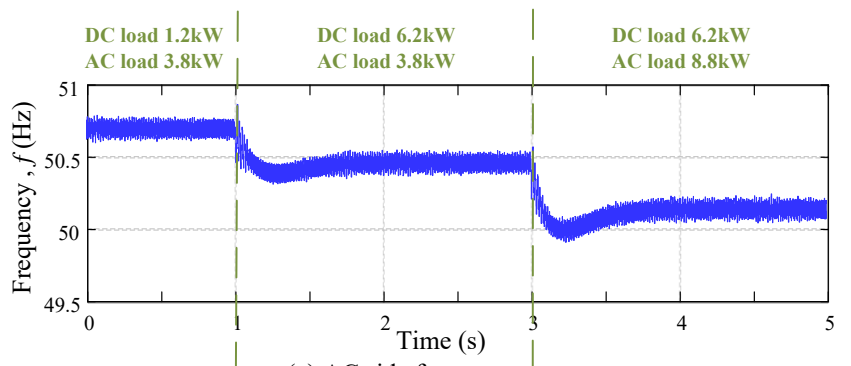

(a) AC side frequency.
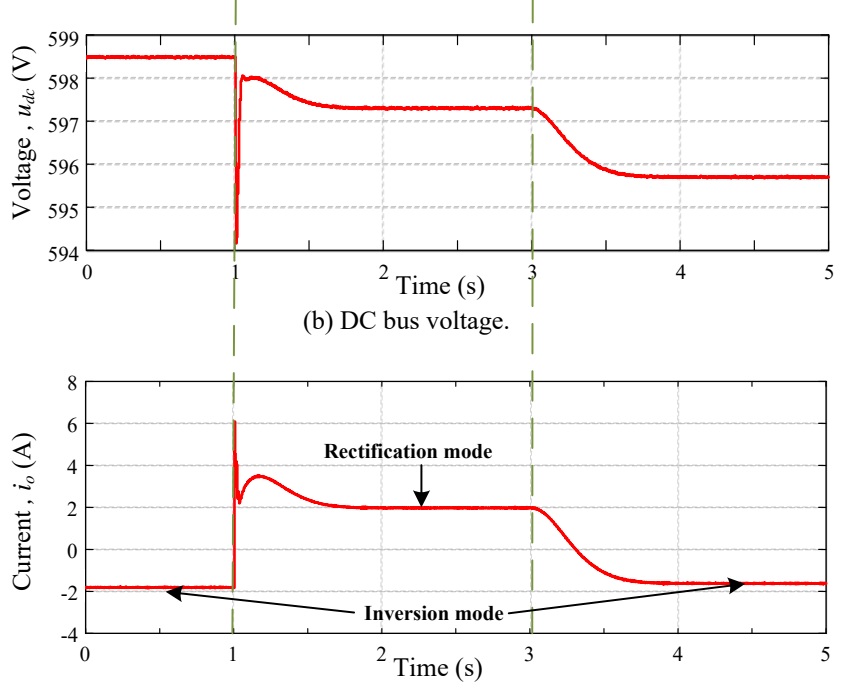

(c) ILC output current to DC side, positive in rectification mode.

Fig. 10. Simulation results of the hybrid microgrid.

\section{B. Improved DC Voltage Stability}

Fig. 12 shows the DC bus voltage under the condition that DC load suddenly increase from light to heavy mode (at $1 \mathrm{~s}$ ) and then decrease back to light mode (at $3 \mathrm{~s}$ ). Without virtual inertial control (blue waveform), $u_{d c}$ waveform oscillate during the DC load sudden changes. However, with the proposed virtual inertia control (red waveform), $u_{d c}$ reaches the steadystate value smoothly whether the load sudden increases or decreases, without any voltage oscillation. Though there is still an inevitable small voltage spike at the moment of load switching, but it returns to the smooth curve quickly. The peak value of the DC voltage spike depends on the value of line impedance or DC capacitor, and is not affected by the value of virtual inertia coefficient $\left(C_{v}\right)$ or virtual damping coefficient $\left(D_{b}\right)$.

It shows that inertia and stability can be improved when the proposed virtual inertia control method is used in ILC. 


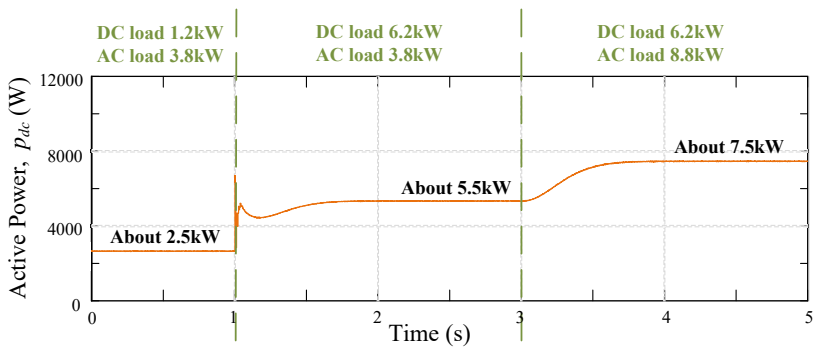

(a) DC subgrid output active power.

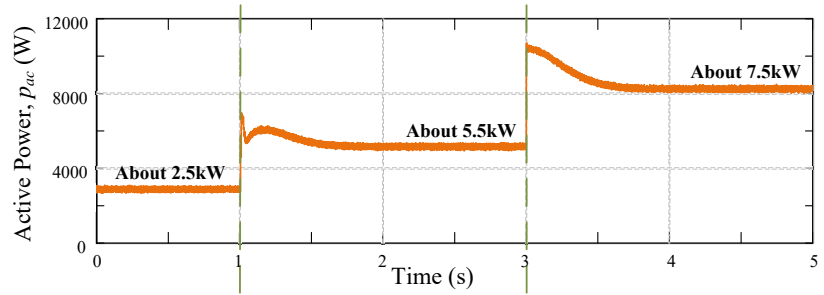

(b) AC subgrid output active power.

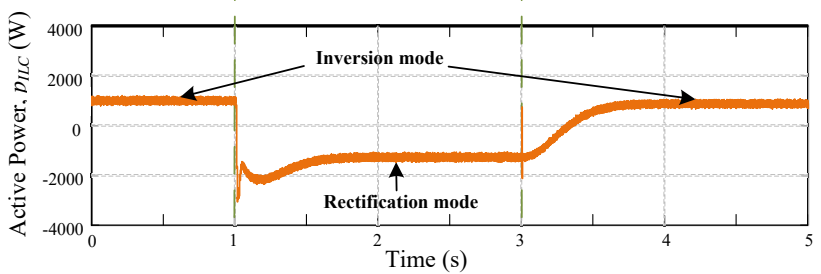

(c) Active power ILC transferred to AC side, positive in invertion mode.

Fig. 11. Simulation results of output active power with virtual inertia control

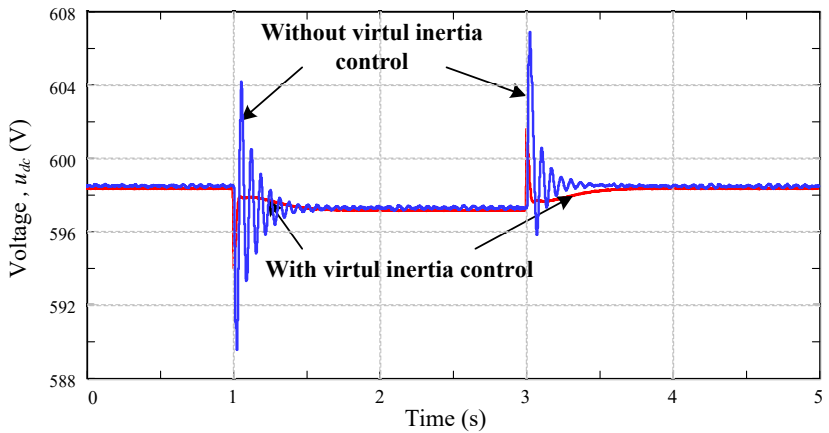

Fig. 12. Simulation results of output active power with virtual inertia control

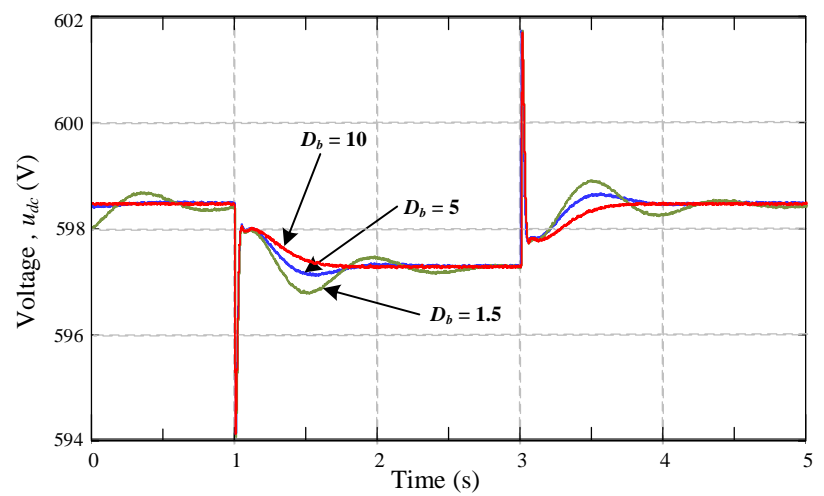

Fig. 13. Simulation results of DC output voltage with $C_{v}=0.00167$ but different $D_{b}$.

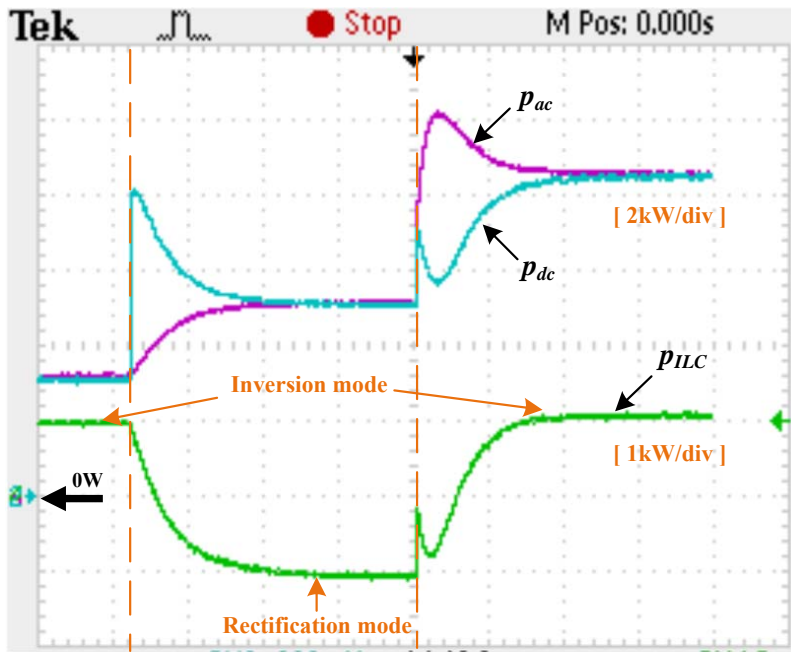

Fig. 14. Experimental results of output active power with virtual inertia control.

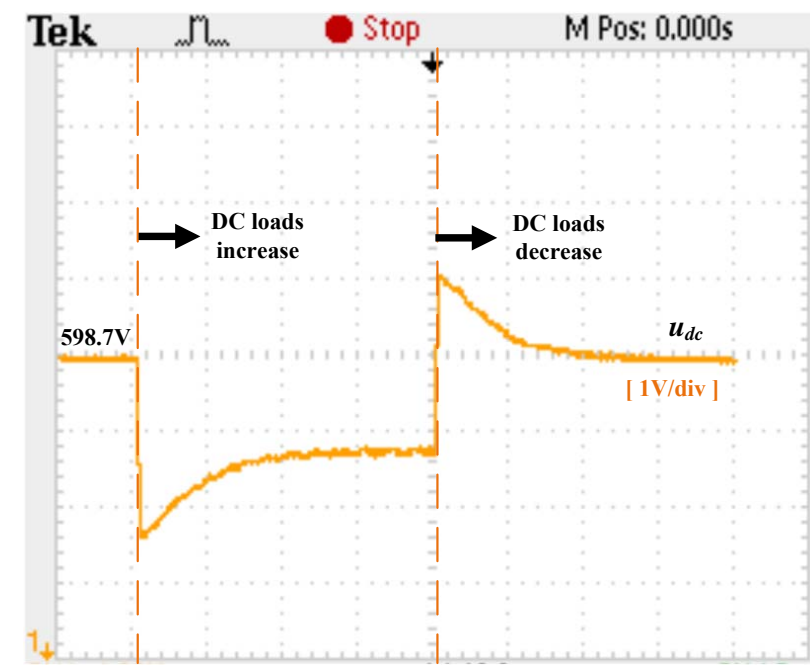

Fig. 15. Experimental results of DC output voltage with proposed virtual inertia control.

In addition, it is worth noting that the influence of virtual damping coefficient $D_{b}$. Fig. 13 reveals the simulation results of DC output voltage when virtual inertia coefficient $C_{v}=$ 0.00167 but virtual damping coefficient $D_{b}$. is different. It can be seen from the three curves that when $C_{v}$ is properly tuned, the value of $D_{b}$ influences the magnitude of fluctuation and the time required to reach steady state. The bigger $D_{b}$ is, the greater the fluctuation is and the slower the steady state is reached.

\section{Experimental Results}

The proposed virtual inertia control method of ILCs is further verified by the experiment results on OPAL-RTLAB real-time digital simulator platform. The hybrid microgrid is built according to Fig. 9 .

Fig. 14 reveals the experimental results of output active power with proposed virtual inertia control under the same conditions as Fig. 11. As can be seen in the picture, from 
Stage 1 to Stage 3, active power of AC side $\left(p_{a c}\right)$ and active power of DC side $\left(p_{d c}\right)$ are approximately equal when reaching steady state. And the active power of ILC $\left(p_{I / ! L / ! C}\right)$ equals to the power shortage of each side. It verified the effectiveness of the proportional power sharing function of the ILC.

In addition, Fig. 15 gives the experimental results of DC output voltage with proposed virtual inertia control under the same conditions as Fig. 12. DC voltage achieves its steady states smoothly after DC side loads sudden change, and no oscillation occurs. It proves that by introducing direct DC voltage control and virtual inertia, system inertia increases and the stability DC voltage is improved.

\section{CONCLUSION}

In this paper, a communication-less virtual inertia control scheme of ILCs for AC/DC hybrid microgrid is presented. The proposed control scheme can increase the DC bus voltage inertia and stability during DC-side load transients and distributed energy fluctuations. Compared to the conventional normalized droop control method, a direct DC voltage control is adopted to realize proportional power sharing and applies virtual inertia technology to ILCs to increase system inertia. Finally, the effectiveness of the control scheme has been verified by the simulation and experimental results.

\section{REFERENCES}

[1] F. Nejabatkhah and Y. W. Li, "Overview of power management strategies of hybrid AC/DC microgrid," IEEE Transactions on Power Electronics, vol. 30, no. 12, pp. 7072-7089, Dec. 2015.

[2] S. M. Malik, X. Ai, Y. Sun, C. Zhengqi and Z. Shupeng, "Voltage and frequency control strategies of hybrid AC/DC microgrid: A review," IET Generation, Transmission and Distribution, vol. 11, no. 2, pp. 303-313, 2612017.

[3] G. Qi, A. Chen and J. Chen, "Improved control strategy of interlinking converters with synchronous generator characteristic in islanded hybrid AC/DC microgrid," CPSS Transactions on Power Electronics and Applications, vol. 2, no. 2, pp. 149-158, June 2017.

[4] A. Mohamed, V. Salehi and O. Mohammed, "Real-time energy management algorithm for mitigation of pulse loads in hybrid microgrids," IEEE Transactions on Smart Grid, vol. 3, no. 4, pp. 1911-1922, Dec. 2012.

[5] M. Hosseinzadeh and F. R. Salmasi, "Robust optimal power management system for a hybrid AC/DC micro-Grid," IEEE Transactions on Sustainable Energy, vol. 6, no. 3, pp. 675-687, July 2015.

[6] P. C. Loh, D. Li, Y. K. Chai and F. Blaabjerg, "Autonomous operation of hybrid microgrid with AC and DC subgrids," IEEE Transactions on Power Electronics, vol. 28, no. 5, pp. 2214-2223, May 2013.

[7] P. C. Loh, D. Li, Y. K. Chai and F. Blaabjerg, "Autonomous control of interlinking converter with energy storage in hybrid ACCDC microgrid," IEEE Transactions on Industry Applications, vol. 49, no. 3, pp. 13741382, May-June 2013.

[8] J. Wang, C. Jin and P. Wang, "A uniform control strategy for the interlinking converter in hierarchical controlled hybrid AC/DC Microgrids," IEEE Transactions on Industrial Electronics, vol. 65, no. 8, pp. 61886197, Aug. 2018.

[9] W. Wu et al., "A virtual inertia control strategy for DC microgrids analogized with virtual synchronous machines," IEEE Transactions on Industrial Electronics, vol. 64, no. 7, pp. 6005-6016, July 2017.
[10] Q. Zhong and G. Weiss, "Synchronverters: Inverters that mimic synchronous generators," IEEE Transactions on Industrial Electronics, vol. 58, no. 4, pp. 1259-1267, April 2011.

[11] Q. Zhong, P. Nguyen, Z. Ma and W. Sheng, "Self-synchronized synchronverters: Inverters without a dedicated synchronization unit," IEEE Transactions on Power Electronics, vol. 29, no. 2, pp. 617-630, Feb. 2014.

[12] A. Fathi, Q. Shafiee and H. Bevrani, "Robust frequency control of microgrids using an extended virtual synchronous generator" IEEE Transactions on Power Systems, vol. 33, no. 6, pp. 6289-6297, Nov. 2018.

[13] H. Alrajhi Alsiraji, A. A. A. Radwan and R. El-Shatshat, "Modelling and analysis of a synchronous machine-emulated active intertying converter in hybrid AC/DC microgrids," IET Generation, Transmission and Distribution, vol. 12, no. 11, pp. 2539-2548, 1962018.

[14] J. Alipoor, Y. Miura and T. Ise, "Stability assessment and optimization methods for microgrid with multiple VSG units," IEEE Transactions on Smart Grid, vol. 9, no. 2, pp. 1462-1471, March 2018.

[15] J. Liu, Y. Miura and T. Ise, "Comparison of dynamic characteristics between virtual synchronous generator and droop control in inverterbased distributed generators," IEEE Transactions on Power Electronics, vol. 31, no. 5, pp. 3600-3611, May 2016.

[16] X. Lu, J. M. Guerrero, K. Sun and J. C. Vasquez, "An improved droop control method for DC microgrids based on low bandwidth communication with DC bus voltage restoration and enhanced current sharing accuracy," IEEE Transactions on Power Electronics, vol. 29, no. 4, pp. 1800-1812, April 2014.

[17] D. Chen, Y. Xu and A. Q. Huang, "Integration of DC microgrids as virtual synchronous machines into the AC grid," in IEEE Transactions on Industrial Electronics, vol. 64, no. 9, pp. 7455-7466, Sept. 2017. 